\title{
Lithotripter Shockwave-Induced Enhancement of Mouse Melanoma Lung Metastasis: Dependence on Cavitation Nucleation
}

\author{
DOUGLAS L. MILLER, Ph.D., CHUNYAN DOU, M.D., and JIANMING SONG, M.D.
}

\begin{abstract}
Purpose: To confirm a previous report of metastasis enhancement by lithotripter shockwaves (LSW) and to test the hypothesis that this effect is attributable to cavitation.

Materials and Methods: The metastatic B16-D5 melanoma cell line was implanted on the hind legs of female C57/b16 mice 12 days before tumor treatment. The tumors were treated with $500 \mathrm{LSW}$ in a waterbath arrangement. The effect of augmented cavitation nucleation was tested by intratumor injection of air bubbles or ultrasound contrast agent gas bodies (UCAGB). The primary tumor was surgically removed on day 1 after treatment. The six groups of mice were sham, LSW, sham + air bubbles, LSW + air bubbles, sham + UCAGB, and LSW + UCAGB. Data were collected for the 113 mice that survived at least 25 days. Lung evaluations were performed blind after 2 weeks of bleaching in Fekete's solution.

Results: The outcomes of the three sham groups were very similar and indicated that the simple injection of material into the tumor did not increase metastasis. In comparison with the pooled shams, both the LSW + air bubbles and LSW + UCAGB groups had statistically significant increases in metastasis counts. Only the LSW + UCAGB group had a significant increase in incidence of metastasis relative to the pooled shams. The LSW + UCAGB also had significantly reduced survival.

Conclusion: Shockwaves can enhance metastasis from tumors, and this effect is attributable to cavitation.
\end{abstract}

\section{INTRODUCTION}

$\mathbf{L}$ ITHOTRIPTER SHOCKWAVES (LSW) have a broad potential for nonthermal biologic effects. ${ }^{1,2}$ These can occur directly, as in stress fractures of kidney stones, or indirectly, via the cavitation mechanism. Cavitation appears to be responsible for adverse side effects of shockwave lithotripsy, and these can be reduced by treatment modifications that reduce cavitation. ${ }^{3}$ The broad potential for nonthermal bioeffects has led to research on the use of LSW for therapeutic applications other than stone disease.

An initially promising application was to cancer therapy. Shockwaves have been shown to have a strong antitumor effect either alone ${ }^{4,5}$ or in combination with other measures such as chemotherapy ${ }^{6,7}$ or gene therapy. ${ }^{8}$ One reported adverse effect of this treatment is a tendency for an increase in metastasis with treatment of highly malignant tumors. ${ }^{9}$ Other shockwave tumor treatment studies have not reported increased metastasis, although these studies were not specifically designed to search for metastases.

The goals of the present study were to confirm the report of metastasis enhancement ${ }^{9}$ in a different tumor model and to test the hypothesis that this effect is attributable to cavitation. The highly metastatic D5 melanoma cell line was used, which metastasizes to the lung in vivo. Tumor treatment was conducted with LSW alone or with augmentation of cavitation activity by intratumor injection of cavitation nuclei, which we have found to be important for reliable cavitation nucleation in vivo. ${ }^{10}$ The results demonstrate enhancement of metastasis by shockwave cavitation.

\section{MATERIALS AND METHODS}

All animal research was conducted with the approval of the University Committee for the Use and Care of Animals and the 
Table 1. Overall Outcome for Treatment Groups

\begin{tabular}{|c|c|c|c|c|c|c|}
\hline & \multirow[b]{2}{*}{ Total } & \multicolumn{2}{|c|}{ Died $<4 d$} & \multirow{2}{*}{$\begin{array}{l}\text { Tumor } \\
\text { recurrence }\end{array}$} & \multirow{2}{*}{$\begin{array}{l}\text { 25-day } \\
\text { survival }\end{array}$} & \multirow{2}{*}{$\begin{array}{l}\text { 42-day } \\
\text { survival }\end{array}$} \\
\hline & & Treatment & Surgery & & & \\
\hline Sham & 19 & 0 & 0 & 2 & 19 & 11 \\
\hline LSW & 21 & 1 & 2 & 5 & 18 & 13 \\
\hline Sham + air bubbles & 21 & 1 & 0 & 0 & 20 & 13 \\
\hline LSW + air bubbles & 21 & 1 & 0 & 7 & 20 & 9 \\
\hline SHAM + UCAGB & 20 & 0 & 0 & 3 & 20 & 12 \\
\hline LSW + UCAGB & 24 & $7^{\mathrm{a}}$ & 1 & 3 & 16 & 3 \\
\hline
\end{tabular}

${ }^{\mathrm{a}} p<0.5$ relative to sham $+\mathrm{UCAGB}$.

guidance of the Unit for Laboratory Animal Medicine of the University of Michigan. For this study, the highly metastatic B16-D5 melanoma cell line was used with female C57/b16 mice. These cells form visible lung metastases about 18 days after tail vein injection and also will grow as subcutaneous (SQ) tumors (as for the B16 melanoma model). The experimental plan utilized established methods to test for enhanced lung metastasis resulting from therapeutic or diagnostic manipulation of tumors implanted on the hind leg. ${ }^{9,11-13}$ A suspension of 2 million cells in $0.1 \mathrm{~mL}$ was injected SQ on the right hind leg of each mouse under ether anesthesia. Treatment was applied after 12 days of tumor growth.

For treatment, mice were weighed (averaging $21 \pm 1.2 \mathrm{~g}$ ) and anesthetized with an intraperitoneal injection of ketamine $75 \mathrm{mg} / \mathrm{kg}$ (Ketaset; Aveco Co., Fort Dodge, IA) and xylazine 15 mg/kg (Rompun; Mobay Corp., Shawnee, KS). The tumor area was shaved and depilated, and the volume of each tumor was estimated using digital calipers to measure the three major axes and calculating the ellipsoidal volume, which averaged $302 \pm 118 \mu \mathrm{L}$. In one third of the mice, a volume of air equal to $10 \%$ of the tumor volume was mixed by hand agitation with an equal volume of saline to form an air bubble suspension, and this was injected into the tumor (IT) with a 28-gauge needle one time approximately 5 minutes prior to LSW treatment. Alternatively, one third of the mice receive an injection of Optison ${ }^{\circledR}$ ultrasound contrast medium at $10 \%$ of tumor volume, the contrast being gently mixed with saline and injected in the same way. The air bubbles and the ultrasound contrast agent gas bodies (UCAGB) served as cavitation nuclei, as described previously. ${ }^{10,14}$ Optison ${ }^{\circledR}$ was purchased from Amersham Health Inc. (Princeton, NJ). According to the package insert, this agent contains 500 to $800 \times 10^{6}$ perfluoropropane gas bodies per milliliter with a mean diameter of 2 to $4.5 \mu \mathrm{m}$. After resuspension, the agent was withdrawn from the sealed vial using an Optispike $^{\mathrm{TM}}$ dispensing pin. A fresh vial was used each treatment day. The anesthetized mouse was mounted on a plastic board, which incorporated a $2.0-\mathrm{cm}$ beam hole surrounded by a foamplastic shield. This mounting was arranged to center the tumor in the beam hole and then placed in a $37^{\circ} \mathrm{C}$ waterbath for exposure as described previously. ${ }^{10}$ After LSW treatment, the mice were removed from the bath, dried, and allowed to recover in warmed chambers before return to regular cages.

A laboratory LSW system, which was similar to a Dornier HM3 lithotripter and fitted with standard spark gaps (Dornier Medical Systems, Kennesaw, GA), was used for treatment as described previously. ${ }^{10}$ Briefly, the water in the exposure bath was degassed for 1 hour by vacuum prior to filling of the bath and then continuously filtered to minimize the occurrence of cavitation. The LSW output was measured at the focus, $12 \mathrm{~cm}$ from the mouth of the reflector, and the main pulse had a spatial peak pressure amplitude of $42.6 \pm 1.4 \mathrm{MPa}$ peak positive
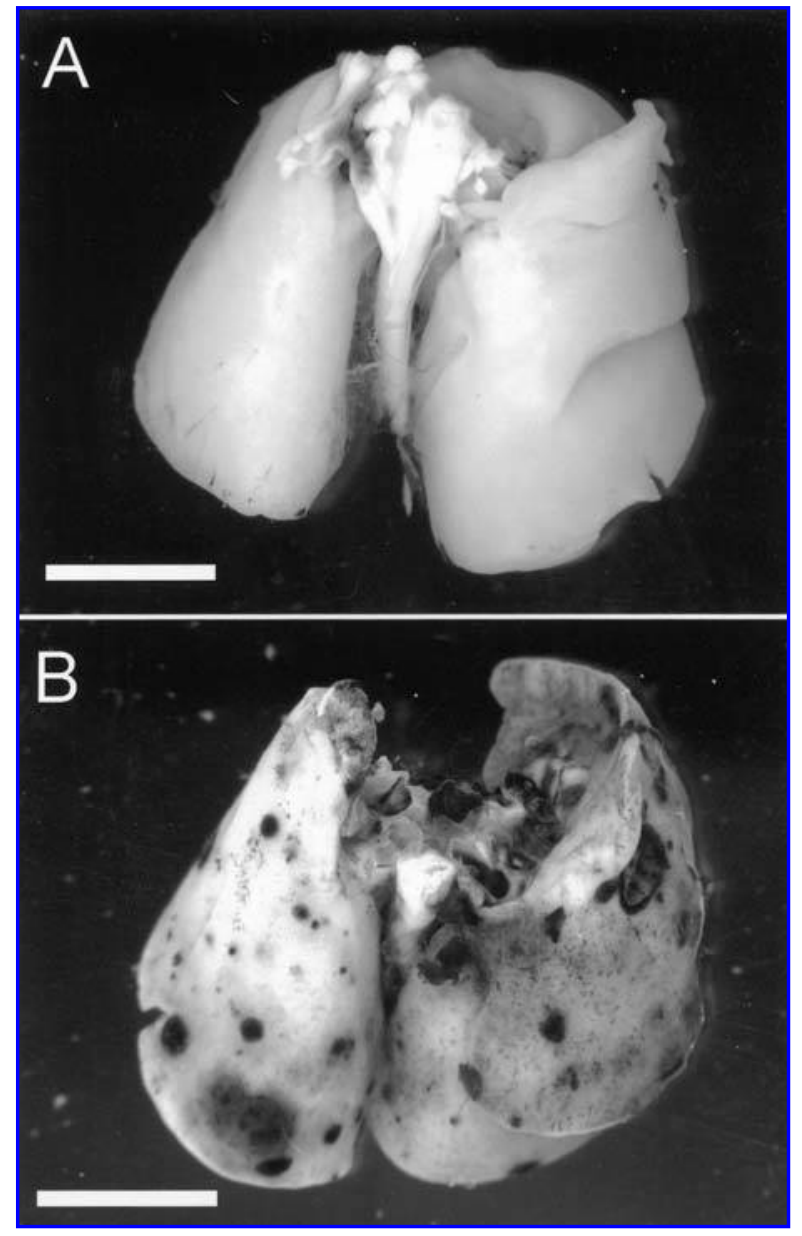

FIG. 1. Mouse lungs after (A) sham + UCAGB and (B) 500 LSW + UCAGB. Dark spots, which appear mostly near surface of lungs in panel B, are melanoma metastases, which were scored as zero in (A) and 13 in (B). 
Table 2. Lung Metastasis Occurrence and Counts in Mice Surviving at Least 25 Days

\begin{tabular}{lcccc}
\hline & $\begin{array}{c}25 \text {-day } \\
\text { survival }\end{array}$ & $\begin{array}{c}\text { No. with metastasis } \\
(\%)\end{array}$ & $\begin{array}{c}\text { Metastases } \\
\text { count (SEM) }\end{array}$ & Count $\mathrm{p}$ values \\
\hline Sham & 19 & $10(53)$ & $1.7(0.7)$ & 0.77 \\
LSW & 18 & $9(50)$ & $3.4(1.2)$ & 0.93 \\
Sham + air bubbles & 20 & $11(55)$ & $2.5(0.7)$ & 0.24 \\
LSW + air bubbles & 20 & $15(75)$ & $3.5(0.8)$ & $<.046$ \\
SHAM + UCAGB & 20 & $12(60)$ & $2.0(0.8)$ & $<0.001$ \\
LSW + UCAGB & 16 & $15(94)^{\mathrm{a}}$ & $8.2(1.2)$ & $<0.001$ \\
\hline
\end{tabular}

${ }^{\mathrm{a}} p<0.05$ relative to pooled shams (i.e., 33 of 59 or $55 \%$ ).

and $-7.4 \pm 1.9 \mathrm{MPa}$ peak negative. For tumor treatment, 500 $\mathrm{LSW}$ were delivered at a $2-\mathrm{Hz}$ rate.

After treatment, sufficient time must be allowed for any cells metastasizing to the lungs to grow into tumors of visible size. To permit prolonged survival, the primary tumor was surgically removed the day after treatment. If the primary tumor is not surgically removed, it will continue to grow and kill the mice within about 10 days, which is too brief a time to allow formation of visible lung metastases. After the mice were reanesthetized, excision was accomplished by removal of the leg at the hip and suture of the skin over the area. Recovery was aided by injection of $1 \mathrm{~mL}$ of warm saline intraperitoneally, together with buprenorphine analgesic $0.1 \mathrm{mg} / \mathrm{kg} \mathrm{SQ}$.

The experimental plan was for 6 groups of 20 mice each, with the overall outcome listed in Table 1 . This moderately large group size was needed for statistical validity, owing to the background metastasis rate. The groups were sham, LSW, sham + air bubbles, LSW + air bubbles, sham + UCAGB, and LSW + UCAGB. Mice were treated on each of 2 days per week for 6 weeks. Normally, one or two extra mice were available each day to replace mice that failed to grow injectable tumors or died at the time of treatment. On one treatment day, only nine mice were available, and a sham exposure was omitted from the study. A total of 126 mice were treated. Ten mice died within 1 day of treatment, which was attributed to the exposure procedure. Three other mice died of complications from the surgery. The mice were euthanized if a tumor recurred and reached $3000 \mu \mathrm{L}$ in volume or at 42 days after treatment. Data were collected for the 113 mice that survived at least 25 days. For evaluation, the lungs were removed and bleached in Fekete's solution to count the pigmented lung metastases. ${ }^{12}$ The metastatic nodules were evaluated blind after 2 weeks in the Fekete's solution by examination under a lowpower stereo microscope. Figure 1 shows the gross lung photographs from a sham + UCAGB mouse and from an LSW + UCAGB mouse. Statistical comparisons of the mean metastases counts were made using the Mann-Whitney rank sum test, and the occurrence rates were compared using the $\mathrm{z}$ test (SigmaStat 2.0; SPSS Inc., Chicago, IL).

\section{RESULTS}

The D5 melanoma proved to be highly metastatic, with a lung metastasis rate of $53 \%$ in sham-treated animals, and a recurrence rate of the primary tumor of $16 \%$ at the site of the surgery (Table 2). The results in the three sham groups were very similar and indicate that the simple injection of material into the tumor did not increase metastasis. For comparison between sham and treated groups, only the LSW + UCAGB group had a statistically significant increase in metastases (Fig. 2). In comparison with the pooled shams, both the air bubbles + LSW and LSW + UCAGB groups had statistically significant increases in metastasis counts, but only the LSW + UCAGB group had a significant increase in the occurrence of metastasis relative to the pooled shams.

The biologic effects generated by the LSW + UCAGB included reduced survival. Only 3 of the 23 animals in this group survived to the 42-day cut-off, and 7 died soon after the treatment (i.e., within 1 day) (see Table 1 ). The high early death rate (7 of 23), which was apparently attributable to the treatment, was significantly increased relative to the sham + UCAGB group ( 0 of 20$)(P=0.024)$ and to the pooled shams (1 of 60) $(P=0.005)$.

\section{DISCUSSION}

The goals of this study were to confirm the report of an enhancement of metastasis for LSW tumor treatment, ${ }^{9}$ and to test the hypothesis that the effect is attributable to cavitation. The shockwave treatment by itself produced a nonsignificant in-

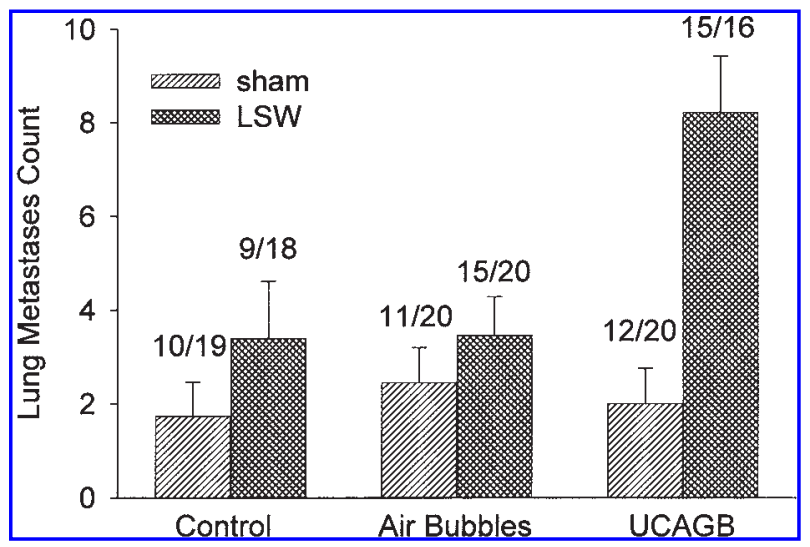

FIG. 2. Comparison of mean lung metastasis count for each group with standard error bar. Fractional number of mice surviving at least 25 days with lung metastasis is shown above each bar. 
crease in metastasis counts. The background count was relatively high in this study, which reduces the power of this negative finding. With enhanced cavitation nucleation, a significant increase in metastasis counts was found. This was particularly strong for nucleation with UCAGB, which gave an increase in the rate of metastasis occurrence. Therefore, although the LSW alone failed to increase metastasis significantly, the results should be considered a confirmation of the positive report ${ }^{9}$ : Treatment of tumors with LSW can increase metastatic spread of cancer. The hypothesis that the metastasis enhancement effect is attributable to cavitation was confirmed in this study. Enhancing cavitation nucleation increased metastasis, and the greater nucleation provided by the numerous gas bodies in Optison was most efficacious compared with LSW or LSW + air bubbles.

Only three mice appeared to succumb to complications of the surgery. The survival was reduced in the LSW + UCAGB group compared with other groups, apparently as a result of the treatment. In this group, blood was usually visible under the skin around the tumor after treatment, which suggests that hemorrhage contributed to the lethal effect. This agrees with previous findings of reduced survival of mice treated with IV-injected contrast agent and LSW exposure to the abdomen ${ }^{15}$ and with IV-injected contrast agent and LSW tumor treatment. ${ }^{10}$ The high rate of metastasis found in this study indicates a substantial release of tumor cells or debris into the circulation, which may also contribute to the lethal effect (e.g., by pulmonary embolism).

Both the metastasis enhancement effect and the lethality result from LSW cavitation. Cavitation involves the growth of giant cavities during and after the prolonged rarefactional phase of the shockwave pulse, ${ }^{16,17}$ which can rupture 200 $\mu \mathrm{m}$-diameter tubes. ${ }^{18}$ However, the vessel rupture leading to hemorrhage and metastasis need not originate in the blood. Previous work has shown that cavitation bubbles can be imaged in shockwave-exposed tissue above a threshold of only 1.5 to $3.5 \mathrm{MPa} .{ }^{19}$ In contrast, bubbles were not detected in circulating blood for full-amplitude LSW $(\sim-10 \mathrm{MPa})$ owing to the continuous filtering in vivo, which depletes cavitation nuclei in blood. ${ }^{20}$ In this present work, the nuclei were injected into the tumor tissue (rather than the blood) and were effective in inducing hemorrhage and enhancing metastasis. Thus, it seems likely that the nucleation of cavitation giving the adverse consequences of LSW occurs primarily in the tissue interstitium.

\section{CONCLUSION}

Lithotripter shockwaves can enhance metastasis from tumors, and this effect is attributable to the cavitation mechanism. The particularly strong effects induced by the presence of UCAGB support a recommendation that lithotripsy not be performed within 24 hours after ultrasound contrast agent administration. ${ }^{21}$ Furthermore, given the findings of metastasis enhancement, it appears that LSW should not have a role in cancer therapy and should be avoided in patients with malignancy within the lithotripsy-treatment volume.

\section{ACKNOWLEDGMENTS}

We thank Dr. A.E. Chang of the University of Michigan Comprehensive Cancer Center, for providing the D5 mouse melanoma cell line. This work was supported by U.S. Public Health Service grants EB002782 and EB00338 awarded by the National Institutes of Health.

\section{REFERENCES}

1. Delius M. Medical applications and bioeffects of extracorporeal shockwaves. Shockwaves 1994;4:55-72.

2. Lingeman JE, Kim SC, Kuo RL, McAteer JA, Evan AP. Shockwave lithotripsy: Anecdotes and insights. J Endourol 2003;17: 687-693.

3. Evan AP, Willis LR, McAteer JA, et al. Kidney damage and renal functional changes are minimized by waveform control that suppresses cavitation in shock wave lithotripsy. J Urol 2002;168: 1556-1562.

4. Hoshi S, Orikasa S, Suzuki K, Saitoh T, Takahashi T, Yosihikawa K, Kuwahara M, Nose M. High-energy underwater shockwave treatment for internal iliac muscle metastasis of prostatic cancer: A first clinical trial. Jpn J Cancer Res 1995;86:424-428.

5. Gamarra F, Spelsberg F, Dellian M, Goetz AE. Complete local tumor remission after therapy with extra-corporeally applied highenergy shock waves (HESW). Int J Cancer 1993;55:153-156.

6. Hoshi S, Orikasa S, Kawahara M-A, Suzuki K, Shirai S, Yoshikawa K, Nose M. Shockwave and THP-Adriamycin for treatment of rabbit's bladder cancer. Jpn J Cancer Res 1992;83:248-250.

7. Weiss N, Delius M, Gambihler S, Eichholtz-Wirth H, Dirschedle P. Effect of shockwaves and cisplatin on cisplatin-sensitive and -resistant rodent tumors in vivo. Int. J Cancer 1994;58:693-699.

8. Song J, Tata D, Li L, Taylor J, Bao S, Miller DL. Combined shockwave and immunogene therapy of mouse melanoma and renal carcinoma tumors. Ultrasound Med Biol 2002;28:957-964.

9. Oosterhof GO, Cornel EB, Smits GA, Debruyne FM, Schalken. The influence of high-energy shockwaves on the development of metastases. Ultrasound Med Biol 1996;22:339-344.

10. Miller DL, Song J. Lithotripter shockwaves with cavitation nucleation agents produce tumor growth reduction and gene transfer in vivo. Ultrasound Med Biol 2002;28:1343-1348.

11. Kadmon D, Heston WD, Fair WR. Treatment of a metastatic prostate derived tumor with surgery and chemotherapy. J Urol 1982;127:1238-1242.

12. Nathanson SD, Nelson L, Anaya P, Havstad S, Hetzel FW. Development of lymph node and pulmonary metastases after local irradiation and hyperthermia of foot pad melanomas. Clin Exp Metastas 1991;9;377-392.

13. Schreiber S, Gross S, Brandis A, Harmelin A, Rosenbach-Belkin V, Scherz A, Salomon Y. Local photodynamic therapy of rat C6 glioma xenografts with Pd-bacteriopheophorbide leads to decreased metastases and increase of animal cure compared with surgery. Int J Cancer 2002;99:279-285.

14. Miller DL, Kripfgans OD, Fowlkes JB, Carson PL. Cavitation nucleation agents for nonthermal ultrasound therapy. $\underline{\mathrm{J} \text { Acoust Soc }}$ Am 2000;107:3480-3486.

15. Miller DL, Gies RA. Consequences of lithotripter shockwave interaction with gas body contrast agent in mouse intestine. $\mathrm{J}$ Urol 1999;162:606-609.

16. Church CC. A theoretical study of cavitation generated by an extracorporeal shockwave lithotripter. J Acoust Soc Am 1989;86: 215-227.

17. Zhu S, Zhong P. Shockwave-inertial microbubble interaction: A 
theoretical study based on the Gilmore formulation for bubble dynamics. J Acoust Soc Am 1999;106:3024-3033.

18. Zhong P, Lin H, Xi X, Zhu S, Bhogte ES. Shockwave-inertial microbubble interaction: Methodology, physical characterization and bioeffect study. J Acoust Soc Am 1999;105:1997-2008.

19. Coleman AJ, Kodama T, Choi MJ, Adams T, Saunders JE. The cavitation threshold of human tissue exposed to $0.2 \mathrm{MHz}$ pulsed ultrasound: Preliminary measurements based on a study of clinical lithotripsy. Ultrasound Med Biol 1995;21:405-417.

20. Williams AR, Delius M, Miller DL, Schwarze W. Investigation of cavitation in flowing media by lithotripter shockwaves both in vitro and in vivo. Ultrasound Med Biol 1989;15:53-60.
21. American Institute of Ultrasound in Medicine. Mechanical bioeffects from diagnostic ultrasound: AIUM Consensus Statements. J Ultrasound Med 2000;19:67-170.

Address reprint requests to: Douglas Miller, M.D. RM 3315 Kresge III University of Michigan Medical Center 200 Zina Pitcher Place Ann Arbor, MI 48109-0553

E-mail:douglm@umich.edu 
This article has been cited by:

1. Hilary Hancock, Matthew R. Dreher, Nigel Crawford, Claire B. Pollock, Jennifer Shih, Bradford J. Wood, Kent Hunter, Victor Frenkel. 2009. Evaluation of pulsed high intensity focused ultrasound exposures on metastasis in a murine model. Clinical \& Experimental Metastasis . [CrossRef]

2. Timothy L. Hall, Kathleen Kieran, Kimberly Ives , J. Brian Fowlkes, Charles A. Cain , William W. Roberts . 2007. Histotripsy of Rabbit Renal Tissue in Vivo: Temporal Histologic TrendsHistotripsy of Rabbit Renal Tissue in Vivo: Temporal Histologic Trends. Journal of Endourology 21:10, 1159-1166. [Abstract] [PDF] [PDF Plus] 